\title{
Natural convection in a cavity with time-dependent flux boundary
}

\author{
L. Zhou ${ }^{1}$, S. W. Armfield ${ }^{1}$ and W. Lin ${ }^{2}$ \\ ${ }^{1}$ School of Aerospace, Mechanical and Mechatronic Engineering \\ The University of Sydney, Sydney NSW 2006, Australia \\ ${ }^{2}$ College of Science and Engineering \\ James Cook University, Townsville QLD 4811, Australia
}

\begin{abstract}
Numerical simulations have been carried out to investigate the unsteady natural convection flow in a cavity subjected to a sidewall heat flux varying sinusoidally with time. With all walls non-slip and the upper and lower boundaries and one sidewall adiabatic, the heating and cooling on the other sidewall produces an alternating direction natural convection boundary layer that discharges hot fluid to the top and cold fluid to the bottom of the cavity, generating a strong and time-varying thermal stratification in the cavity interior. The thermal stratification is shown to be strongly dependent on the forcing frequency.
\end{abstract}

\section{Keywords}

Natural convection flow; Numerical simulation; Heat transfer.

\section{Introduction}

Natural convection flows occur widely in industrial and environmental applications. Examples are ventilation in buildings, passive cooling in nuclear reactors and melting ice shelves. A canonical model considers fluid in a cavity subjected to differential heating via isothermal [1] or isoflux [2] boundary conditions on the vertical walls. The isothermal case has one sidewall at a temperature higher than the ambient and the other at a temperature lower than the ambient. The isoflux case considers a uniform heat flux heating and cooling on opposing sidewalls. In both cases, a rising/falling boundary layer is produced adjacent to the heated/cooled wall. In the cavity interior, a cavityscale circulation and a linear thermal stratification are generated. Extensive studies have investigated the flow dependency on the Rayleigh number, Prandtl number, aspect ratio of the cavity, with scaling relations developed for constant in time heating/cooling [3].

In recent years, natural convection flows in cavities with timevarying thermal boundary conditions have drawn increasing interest, as in many applications, such as the diurnal heating and cooling of buildings, the thermal boundary condition changes periodically with time. Past studies on the effects of a periodic thermal boundary condition mostly focus on the temperature boundary condition by applying a sinusoidal temperature oscillating around a hot/cold temperature on the wall [4]. In addition to a cavity scale circulation the unsteady temperature boundary condition also generate a secondary circulation associated with a local reverse in the heat transfer, that is from the fluid to the wall during the phase when the wall temperature is greater than the ambient, and from the wall to the fluid when the wall temperature is less than the ambient. One observation is that the horizontal heat transfer can be enhanced by the sinusoidal temperature forcing, with maximum enhancement found at a specific forcing frequency, denoted the resonance frequency. However, a better approximation to the diurnal effect of the solar radiation is a periodic flux boundary condition. Researchers have also applied a square wave heating flux on a sidewall and zero temperature on the opposing wall [5]. A similar resonance phenomenon with enhanced horizontal heat transfer at specific forcing frequency was found for the flux boundary condition. For both the periodic temperature and flux boundary conditions the resonance effect is more distinct for larger Rayleigh number. More recent work has investigated the effect of such unsteady boundary conditions on the thermal performance of buildings $[6,7]$.

Numerical simulations have been carried out for natural convection flow in a cavity subjected to a sinusoidal sidewall heat flux. The flow structure at full development is analysed and the thermal performance quantified by a stratification parameter. The stratification exhibits strong dependency on the forcing frequency, and flow regimes are defined with flow features discussed and compared with those for the temperature boundary condition [8].

\section{Numerical Method}

The flow problem of interest is a water-filled cavity $(\mathrm{Pr}=7.0)$ with height and width of $H$. The cavity has all walls non-slip, and the upper and lower boundaries and the right wall are adiabatic. The left wall is subjected to a spatially uniform flux boundary condition varying sinusoidally with time at frequency $F$. At initiation the fluid temperature is $T_{0}$ and the flow is stationary. The domain and boundary conditions are shown in figure 1.

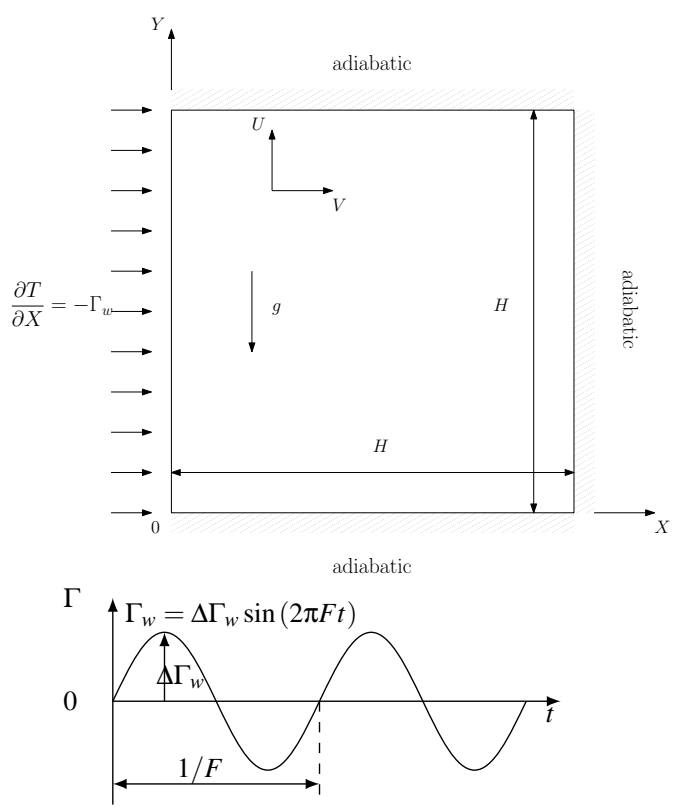

Figure 1. Flow configuration

The governing equations are the incompressible twodimensional Navier-Stokes equations and the temperature 
transport equation, with the Oberbeck-Boussinesq approximation for the buoyancy. Dimensional quantities are non-dimensionalised by the following relations:

$$
\begin{gathered}
(x, y)=\frac{(X, Y)}{H},(u, v)=\frac{(U, V)}{(\alpha / H) R a^{2 / 5}}, \\
\tau=\frac{\alpha R a^{2 / 5}}{H^{2}} t, \theta=\frac{T-T_{0}}{\Delta \Gamma_{w} H}
\end{gathered}
$$

where $x$ and $y$ are the non-dimensional horizontal and vertical coordinate directions, $u$ and $v$ the non-dimensional horizontal and vertical velocities, $\tau$ the non-dimensional time, and $\theta$ the non-dimensioanl temperature. The Rayleigh number and Prandtl number are defined as $R a=g \beta \Delta \Gamma_{w} H^{4} / v \alpha$ and $P r=$ $v / \alpha$, with $g$ the gravity, $\beta$ the coefficient of thermal expansion, $v$ the kinematic viscosity, and $\alpha$ the thermal diffusivity. After the non-dimensionalisation, the start up time for the boundary layer is $\tau \cong 1.0$ and the vertical velocity is $v \cong 1.0$ [9]. The non-dimensional governing equations are

$$
\begin{aligned}
\frac{\partial u}{\partial \tau}+u \frac{\partial u}{\partial x}+v \frac{\partial v}{\partial y} & =-\frac{\partial p}{\partial x}+\frac{\operatorname{Pr}}{\operatorname{Ra}^{\frac{2}{5}}}\left(\frac{\partial^{2} u}{\partial x^{2}}+\frac{\partial^{2} u}{\partial y^{2}}\right) \\
\frac{\partial v}{\partial \tau}+u \frac{\partial v}{\partial x}+v \frac{\partial v}{\partial y} & =-\frac{\partial p}{\partial y}+\frac{\operatorname{Pr}}{R a^{\frac{2}{5}}}\left(\frac{\partial^{2} v}{\partial x^{2}}+\frac{\partial^{2} v}{\partial y^{2}}\right) \\
+P r R a^{\frac{1}{5}} \theta & \frac{\partial u}{\partial x}+\frac{\partial v}{\partial y}=0 \\
\frac{\partial \theta}{\partial \tau}+u \frac{\partial \theta}{\partial x}+v \frac{\partial \theta}{\partial y} & =\frac{1}{R a^{\frac{2}{5}}}\left(\frac{\partial^{2} \theta}{\partial x^{2}}+\frac{\partial^{2} \theta}{\partial y^{2}}\right)
\end{aligned}
$$

The initial and boundary conditions in non-dimensional forms are

$$
\begin{aligned}
& u=v=\theta=0 \text { at } \tau=0 \text { for } 0 \leqslant x \leqslant 1,0 \leqslant y \leqslant 1, \\
& u=v=0, \frac{\partial \theta}{\partial y}=-\sin (2 \pi f \tau) \text { at } x=0 \text { for } 0 \leqslant y \leqslant 1, \tau>0,
\end{aligned}
$$

$$
u=v=0, \frac{\partial \theta}{\partial y}=0 \text { at } y=0 \text { and } y=1 \text { for } 0<x<1, \tau>0 \text {, }
$$

$$
u=v=0, \frac{\partial \theta}{\partial x}=0 \text { at } x=1 \text { for } 0 \leqslant y \leqslant 1, \tau>0,
$$

where $f$ is the non-dimensional forcing frequency $f=$ $\left(H^{2} / \alpha R a^{2 / 5}\right) F$.

The momentum and the temperature transport equations are discretised on a non-uniform non-staggered grid using a fourthorder compact scheme [10]. The advection terms are integrated in time using the second-order Adams-Bashforth method, and the diffusion terms using the deferred correction CrankNicloson method. The linear equations are solved by a Jacobi solver. Continuity is enforced using a fractional step method, and the Possion's pressure correction equation is solved by a Gauss-Seidel solver. The scheme has been validated against published data for the differentially heated cavity [8]. Grid and time dependency tests have also been conducted, comparing the solutions on non-uniform $200 \times 200,400 \times 400$ and $800 \times 800$ grids, with time step $2 \times 10^{-4}$ and $1 \times 10^{-4}$. Using a criteria of a less than $1 \%$ variation in the time averaged stratification, the $200 \times 200$ grid with time step $2 \times 10^{-4}$ and $400 \times 400$ with time step $2 \times 10^{-4}$ have been used for $R a=1 \times 10^{6}$ and $R a=1 \times 10^{8}$, respectively.

\section{Results}

\section{General flow structure and thermal performance}

Subjected to the periodic flux forcing on the left wall, the flow becomes periodic after an initial development. The general flow response in a period at full development is presented in figure 2 for $R a=1 \times 10^{6}$ and $f=0.01$, where $\partial \theta /\left.\partial x\right|_{w}>0$ is denoted the heating phase and $\partial \theta /\left.\partial x\right|_{w}<0$ the cooling phase.

In the first half of the heating phase, the temperature gradient on the boundary rises from zero to its maximum of 1.0. With the increase of temperature gradient, an increasing amount of heat is transferred from the left wall to the cavity interior. After a quarter period since the onset of the heating phase, a rising natural convection boundary layer is formed near the heated wall as shown in figure 2(a), with the temperature on the wall increasing with increasing $y$ location. The hot, positively-buoyant fluid discharged from the boundary layer also forms an intrusion layer immediately below the upper boundary. In the velocity vector plot, the cavity interior is dominated by a cavity-scale clockwise circulation. At $\tau=1046$, the temperature gradient decreases to approximately 0.25 . The hot fluid in the intrusion layer discharged from the boundary layer extends further into the cavity interior, reaches the right wall, and fills the cavity from the top to the bottom. The cavity-scale circulation becomes reverse $\mathrm{L}$ shape at $\tau=1046$ and later breaks into two clockwise circulation, one in the upper region, the other in the lower region of the cavity at $\tau=1050$.

Beyond $\tau=1050$, the temperature gradient becomes negative. The reverse in the direction of the heat flux forcing significantly changes the flow structure in the next four time units (from $\tau=1050$ to 1054 ). Unlike the flow structure for the temperature boundary condition where boundary layer experiences a rising part in the upper region and a falling part in the lower region at this stage [8], the boundary layer for the flux boundary condition is uni-directional. At $\tau=1052$, the increasing heat transfer from the cavity interior to the wall now produces a falling boundary layer in the close-to-wall region. A counterclockwise circulation occurs between the two clockwise circulations near the horizontal midline, with its strength increasing with time, pushing the two clockwise circulations to the upper right and bottom right corners of the cavity. At $\tau=1054$, the counter-clockwise circulation grows to cavity-scale and becomes the primary circulation in the cavity. During this rapid change of the velocity structure of the flow, the temperature structure maintains a stable and approximately linear stratification in the cavity interior, with $\theta \cong 0.2$ near the upper boundary and $\theta \cong 0.05$ near the lower boundary.

In the following cooling phase, a falling boundary layer is fully established at $\tau=1075$, with the temperature on the wall increasing with increasing $y$ location. The cold intrusion discharged from the boundary layer extends to full cavity width. At the end of the period at $\tau=1100$, the lower half of the cavity is filled with $\theta<0$ fluid, and the upper half with $\theta>0$ fluid.

As observed in the temperature contours, the sinusoidal flux boundary condition produces a thermal stratification in the cavity interior at any time in a period. To quantify the strength of the stratification, we use the stratification parameter $S$, defined as the spatially averaged vertical temperature gradient,

$$
S=\int_{0}^{1} \int_{0}^{1} \frac{\partial \theta}{\partial y} d x d y
$$

The time series of $S$ in a period is plotted in figure 3, together with the Nusselt number on the left wall, defined as $N u_{w}=-\left.\int_{0}^{1} \frac{\partial \theta}{\partial x}\right|_{x=0} d y . S$ oscillates at twice the forcing fre- 

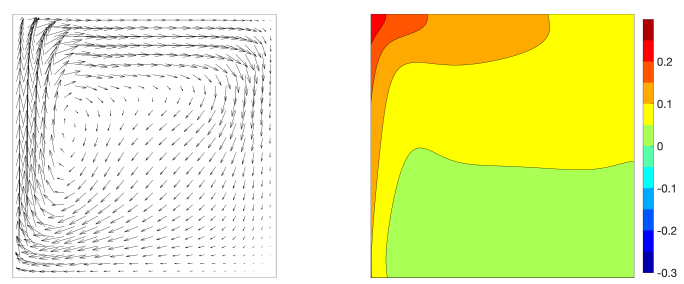

(a) $\tau=1025\left(\partial \theta /\left.\partial x\right|_{w}=1\right)$
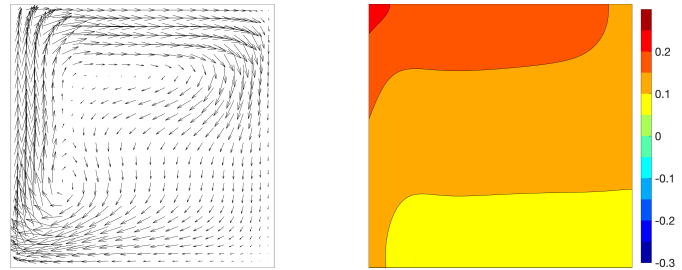

(b) $\tau=1046\left(\partial \theta /\left.\partial x\right|_{w}=0.25\right)$
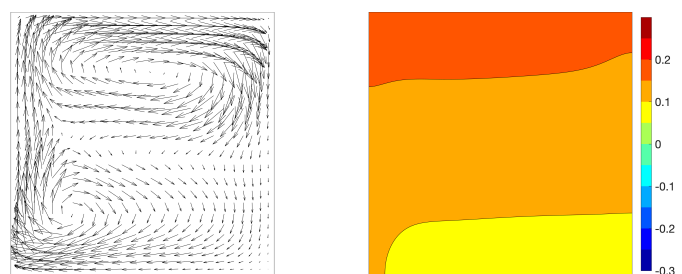

(c) $\tau=1050\left(\partial \theta /\left.\partial x\right|_{w}=0\right)$
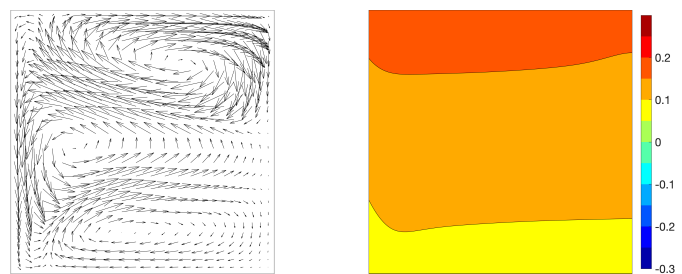

(d) $\tau=1052\left(\partial \theta /\left.\partial x\right|_{w}=-0.13\right)$
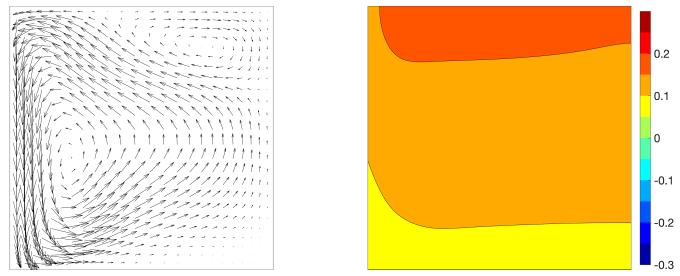

(e) $\tau=1054\left(\partial \theta /\left.\partial x\right|_{w}=-0.25\right)$
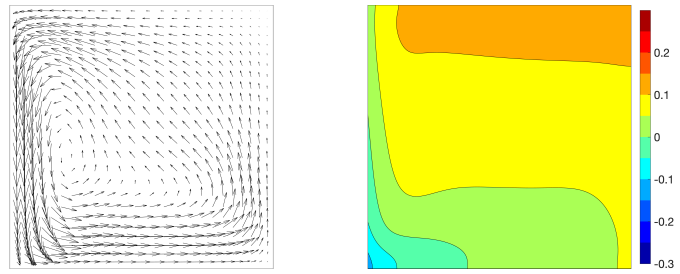

(f) $\tau=1075\left(\partial \theta /\left.\partial x\right|_{w}=-1\right)$
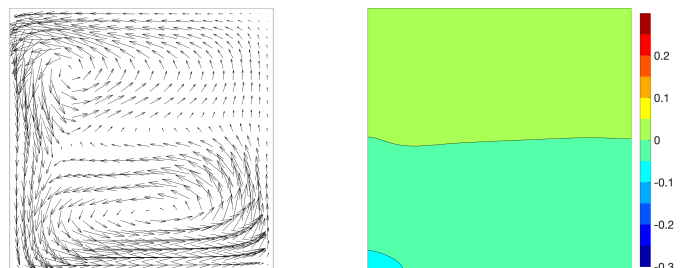

(g) $\tau=1100\left(\partial \theta /\left.\partial x\right|_{w}=0\right)$

Figure 2. Velocity vectors (left) and temperature contours (right) in a period, for $R a=1 \times 10^{6}, f=0.01$.

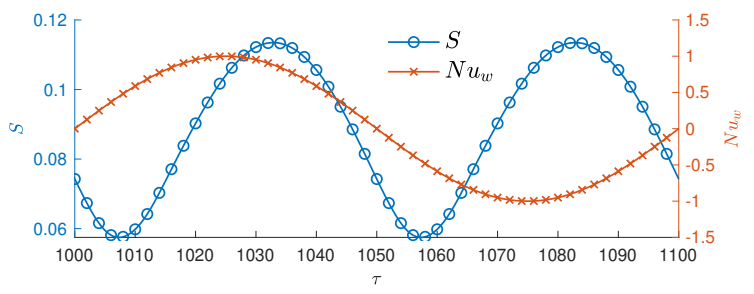

Figure 3. Time series for $S$ and $N u_{w}$, for $R a=1 \times 10^{6}, f=0.01$

quency, $f_{S}=0.02$. The maximum $S$ occurs at approximately $\tau=1033$ and $\tau=1083$, which lags the maximum and minimum forcing fluxes, at $\tau=1025$ and $\tau=1075$, respectively, by approximately $8 \%$ of the period. The time average of $S, \bar{S}$, is 0.088 and the amplitude of the oscillation, $A(S)$, is 0.056 .

\section{Dependency on the forcing frequency}

Further investigations of the thermal performance of the flow show that the stratification has a strong dependency on the forcing frequency. Figure 4 displays the variation of $\bar{S}$ and $A(S)$ with $f$ for $R a=1 \times 10^{6}$, where two distinct regimes have been observed. For $f \gtrsim 0.4$, the time-averaged stratification and the
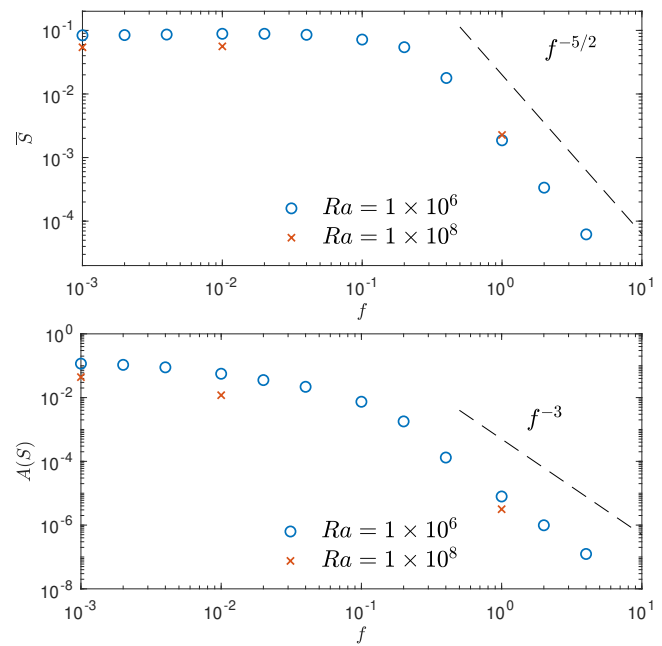

Figure 4. The time average (top) and the amplitude (bottom) of $S$, for $R a=1 \times 10^{6}$.

amplitude of oscillation decreases with increasing forcing frequency, which can be well approximated by $\bar{S} \sim f^{-5 / 2}$ and $A(S) \sim f^{-3}$. This is denoted the high frequency regime. For $f \lesssim 0.4, \bar{S}$ is approximately 0.1 , and $A(S)$ decreases slightly with increasing $f$ with no obvious scaling relation observed, denoted the low frequency regime. Also plotted in figure 4 are results obtained for $R a=1 \times 10^{8}$ showing generally similar behaviour to the $R a=1 \times 10^{6}$ results, although with a small reduction in both $\bar{S}$ and $A(S)$ in the low frequency regime.

The start up time for the isoflux heated natural convection boundary layer is $\Delta \tau \cong 1.0$, and thus for large $f$ the boundary layer will not be fully developed, and will be parallel over most of its height [9]. This can be observed in figure 5, which shows the temperature contours at the end of the heating phase for $f=1$ and 4 . The boundary layer is also thinner and has a larger parallel region for increasing $f$. Similar behaviour was observed for the temperature boundary condition case, when $\bar{S}$ also reduced for large $f$. In the temperature case it was shown that the reduction in $\bar{S}$ was a result of the reduction in the volume of fluid ejected by the boundary layer as the boundary layer width and velocity reduced with increasing $f$ combined with 
a further reduction in the volume of fluid re-entrained by the boundary layer. This led to an $\bar{S} \sim f^{-2}$ relation for the temperature boundary condition case [8]. The flux case shows a more rapid rate of reduction of $\bar{S} \sim f^{-5 / 2}$, and this further rate of reduction is a result of the maximum temperature of the fluid ejected from the rising boundary layer being dependent on the period of the heating phase, which reduces for increasing $f$, whereas for the temperature boundary condition case it is always equal to $\theta=1.0$ (and $\theta=-1.0$ for the falling boundary layer). In figure 5 the maximum temperature is approximately 0.02 for $f=1$, and approximately 0.01 for $f=4$.

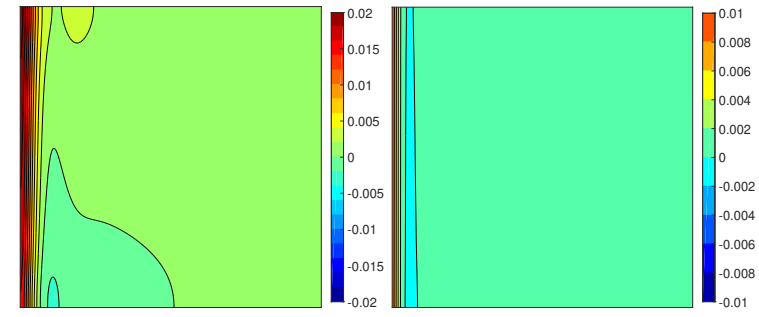

Figure 5. The temperature contours at the end of the heating phase at $f=1$ (left) and $f=4$ (right), for $R a=1 \times 10^{6}$.

In the low frequency regime the relation of $\bar{S}$ to $f$ is quite different to that observed for the temperature boundary condition case, where $\bar{S}$ increases with increasing $f$ [8]. In that case the lower the frequency the closer the temperature of the fluid in the cavity to that on the boundary, which has no vertical variation, and the stratification then reduces with reducing $f$. For the flux boundary condition the temperature on the boundary varies, increasing in the vertical direction. As $f$ is reduced the stratification of the fluid in the cavity approaches that of the boundary temperature, while the overall temperature increases as the increased heating period, for small $f$, means increased total heat transfer. This can be seen in figure 6 where temperature contours for $f=0.001$ and $f=0.01$ are shown.
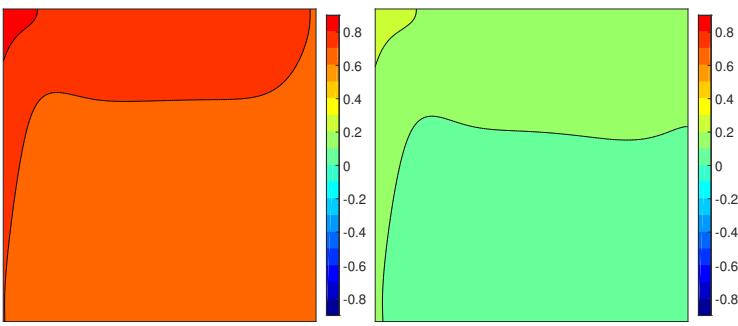

Figure 6. The temperature contours at maximum $S$ at $f=0.001$ (left) and $f=0.01$ (right), for $R a=1 \times 10^{6}$.

\section{Conclusions}

Numerical results have been obtained for the natural convection flow in a cavity subjected to a time-dependent flux boundary condition. The heat flux is sinusoidally varying with time and is applied spatially uniformly on the left sidewall, with other walls adiabatic. The fully developed flow shows a periodic temperature structure, with a rising boundary layer produced in the heating phase and a falling boundary layer in the cooling phase. The cavity interior is mostly dominated by a primary circulation, clockwise in the heating phase and counter-clockwise in the cooling phase, with significant changes happening when the heat transfer at the wall reverses its direction. The stratification of the cavity also changes periodically with time at a frequency double that of the forcing frequency. The time average and the amplitude of the stratification parameter are dependent on the forcing frequency, with $\bar{S}$ approximately constant and $A(S)$ slightly decreasing with increasing $f$ for $f \lesssim 0.4$, and $\bar{S} \sim f^{-5 / 2}$ and $A(S) \sim f^{-3}$ for $f \gtrsim 0.4$. The high frequency regime behaviour of the flow is similar to that for the temperature boundary condition case, although with more rapid decay. In the low frequency regime the flow exhibits different behaviour from that for the temperature boundary condition case, where $\bar{S}$ reduces with reducing $f$. Results obtained at $R a=1 \times 10^{8}$ show largely similar behaviour to the main $R a=1 \times 10^{6}$ results for $\bar{S}$ and $A(S)$, although with a reduction in both $\bar{S}$ and $A(S)$ in the low frequency regime for the higher $R a$.

\section{Acknowledgements}

The authors wish to acknowledge the support of the Australian Research Council and contributions to this work by N. Williamson and M. P. Kirkpatrick.

\section{References}

[1] Catton, I. (1978). Natural convection in enclosures. in Proc. 6th International Heat Transfer Conference, Toronto, Canada, 6, 13-43.

[2] Kimura, S., Bejan, A. (1984). The Boundary Layer Natural Convection Regime in a Rectangular Cavity With Uniform Heat Flux From the Side. Journal of Heat Transfer, 106, 98-103. (DOI:10.1115/1.3246666).

[3] Patterson, J., Imberger, J. (1980). Unsteady natural convection in a rectangular cavity. Journal of Fluid Mechanics, 100, 65-86. (DOI:10.1017/S0022112080001012).

[4] Kwak, H.S., Hyun, J.M. (1996). Natural convection in an enclosure having a vertical sidewall with time-varying temperature. Journal of Fluid Mechanics, 329, 65-88. (DOI:10.1017/S0022112096008828).

[5] Lage, J.L., Bejan, A. (1993). The resonance of natural convection in an enclosure heated periodically from the side. International Journal of Heat and Mass Transfer, 36, 2027-2038. (DOI:10.1016/S0017-9310(05)80134-6).

[6] Saha, S., Patterson, J., Lei, C. (2010). Natural convection and heat transfer in attics subject to periodic thermal forcing. International Journal of Thermal Sciences, 49, 1899 1910. (DOI:10.1016/j.ijthermalsci.2010.05.010).

[7] Zhai, H., Nie, B., Chen, B., Xu F. (2019). Unsteady flows on a roof imposed by a periodic heat flux: 2D simulation and scaling analysis. International Journal of Thermal Sciences, 145, 106002. (DOI:10.1016/j.ijthermalsci.2019.106002).

[8] Zhou, L., Armfield, S.W., Williamson, N., Kirkpatrick, M.P., Lin, W. (2020). Natural convection in a cavity with time-varying thermal forcing on a sidewall. International Journal of Heat and Mass Transfer, 150, 119234. (DOI:10.1016/j.ijheatmasstransfer.2019.119234).

[9] Armfield, S.W., Patterson, J.C., Lin, W. (2007). Scaling investigation of the natural convection boundary layer on an evenly heated plate. International Journal of Heat and Mass Transfer, 50, 1592-1602. (DOI:10.1016/j.ijheatmasstransfer.2006.08.020).

[10] Zhou, L., Armfield, S., 2016. A compact fourthorder spatial discretisation applied to the NavierStokes equations. ANZIAM Journal, 56, 481-501. (DOI:10.21914/anziamj.v56i0.9422). 